PROCEEDINGS OF THE

AMERICAN MATHEMATICAL SOCIETY

Volume 140, Number 1, January 2012, Pages 279-289

S 0002-9939(2011)10970-5

Article electronically published on June 1, 2011

\title{
ALMOST PERIODIC SOLUTIONS TO SOME SECOND-ORDER NONAUTONOMOUS DIFFERENTIAL EQUATIONS
}

\author{
TOKA DIAGANA \\ (Communicated by Yingfei Yi) \\ In memory of my friend and colleague Cora Sadosky
}

\begin{abstract}
This paper is concerned with the existence of almost periodic mild solutions to some second-order equations. Using dichotomy tools and the Schauder fixed point theorem, the existence of almost periodic mild solutions to those second-order evolution equations is established. To illustrate our abstract results, the existence of almost periodic solutions to a damped second-order boundary value problem is also discussed.
\end{abstract}

\section{INTRODUCTION}

Let $\mathbb{H}$ be a separable infinite dimensional complex Hilbert space. Our main objective in this paper is to study the existence of almost periodic solutions to the classes of nonautonomous second-order differential equations

$$
\frac{d^{2} u}{d t^{2}}+a(t) \frac{d u}{d t}+b(t) A u=h(t, u)
$$

where $A: D(A) \subset \mathbb{H} \mapsto \mathbb{H}$ is a self-adjoint linear operator on $\mathbb{H}$ whose spectrum consists of isolated eigenvalues $0<\lambda_{1}<\lambda_{2}<\ldots<\lambda_{l} \rightarrow \infty$ as $l \rightarrow \infty$ with each eigenvalue having a finite multiplicity $\gamma_{j}$ equal to the multiplicity of the corresponding eigenspace, $a, b: \mathbb{R} \mapsto \mathbb{C}$ are almost periodic and $h: \mathbb{R} \times \mathbb{H} \mapsto \mathbb{H},(t, u) \rightarrow h(t, u)$ is almost periodic in $t \in \mathbb{R}$ uniformly in $u \in \mathbb{H}$. For that, we study the existence of almost periodic solutions to the more general second-order differential equations

$$
\frac{d}{d t}\left[\frac{d u}{d t}+g(t, u(t))\right]=w(t) A u(t)+f(t, u(t)), \quad t \in \mathbb{R},
$$

where $A: D(A) \subset \mathbb{H} \mapsto \mathbb{H}$ is the same as in Eq. (1.1), the function $w: \mathbb{R} \mapsto \mathbb{C}$ given by $w(t)=\rho(t) e^{i \theta(t)}$ for all $t \in \mathbb{R}$ is almost periodic and there exist the constants $\rho_{0}, \rho_{1}>0$ and $\theta_{0} \in\left(0, \frac{\pi}{4}\right)$ such that $\rho_{0} \leq \rho(t) \leq \rho_{1}$, and

$$
\pi+2 \theta_{0}<\theta(t)<2 \pi-2 \theta_{0} \text { for all } t \in \mathbb{R},
$$

and the functions $f, g: \mathbb{R} \times \mathbb{H} \mapsto \mathbb{H}$ are almost periodic in the first variable uniformly in the second variable.

Received by the editors November 17, 2010.

2010 Mathematics Subject Classification. Primary 39A24, 42A75, 35L10, 37L05, 34D09.

Key words and phrases. Schauder fixed point theorem, exponential dichotomy, almost periodic, second-order differential equation. 
Our strategy consists of rewriting Eq. (1.2) as a nonautonomous first-order differential equation on $\mathbb{H} \times \mathbb{H}$ and then applying the Schauder fixed point to it with the help of dichotomy tools. Indeed, assuming that $u$ is twice differentiable and setting $z:=\left(u, u^{\prime}+g(t, u)\right)^{T}$, where $T$ denotes the transpose, then Eq. (1.2) can be rewritten on $\mathbb{X}:=\mathbb{H} \times \mathbb{H}$ in the following form:

$$
\frac{d z}{d t}=\mathcal{A}(t) z+F(t, z(t)), t \in \mathbb{R},
$$

where $\mathcal{A}(t)$ is the family of $2 \times 2$-operator matrices defined by

$$
\mathcal{A}(t)=\left(\begin{array}{cc}
0 & I_{\mathbb{H}} \\
& \\
w(t) A & 0
\end{array}\right), \quad t \in \mathbb{R},
$$

where $I_{\mathbb{H}}$ is the identity operator of $\mathbb{H}$.

Clearly, the domain $D(\mathcal{A}(t))=D(A) \times \mathbb{H}$ is constant in $t \in \mathbb{R}$.

Notice that the term $F$ appearing in Eq. (1.4) is defined on $\mathbb{R} \times \mathbb{X}_{\alpha}$ for some $\alpha \in$ $(0,1)$ by $F(t, z(t))=(-g(t, u), f(t, u))^{T}$, where $\mathbb{X}_{\alpha}$ is the real interpolation space of order $(\alpha, \infty)$ between $\mathbb{X}$ and $D(\mathcal{A}(t))$ given by $\mathbb{X}_{\alpha}=(\mathbb{X}, D(\mathcal{A}(t)))_{\alpha, \infty}=\mathbb{H}_{\alpha} \times \mathbb{H}$, with $\mathbb{H}_{\alpha}:=(\mathbb{H}, D(A))_{\alpha, \infty}$.

Now, assuming that $a: \mathbb{R} \mapsto \mathbb{C}$ is differentiable, one can easily see that Eq. (1.1) is a special case of Eq. (1.2). Indeed, Eq. (1.1) can be rewritten as

$$
\frac{d}{d t}\left[\frac{d u}{d t}+\widetilde{g}(t, u(t))\right]=\widetilde{w}(t) A u(t)+\widetilde{f}(t, u(t)), \quad t \in \mathbb{R},
$$

where $\widetilde{w}(t)=-b(t), \widetilde{g}(t, u)=a(t) u$, and $\widetilde{f}(t, u)=h(t, u)+u a^{\prime}(t)$ for all $t \in \mathbb{R}$.

Let us mention that particular cases of Eq. (1.2) have been considered in the literature; see, e.g., 1, 2, 3, 11, 12, 13. Recently, the existence of solutions to Eq. (1.2) when $w(t)=1$ for all $t \in \mathbb{R}$ was studied and obtained in [8. However, to the best of our knowledge, the original problem which consists of the existence of almost periodic mild solutions to both Eq. (1.2) and Eq. (1.1) remains an untreated question, which constitutes the main motivation of this paper. We essentially make extensive use of ideas of [4, 6, 7, 9] and dichotomy tools, as well as the Schauder fixed point theorem to obtain our existence results.

\section{Preliminaries}

Let $(\mathbb{X},\|\cdot\|)$ be a Banach space. If $L$ is a linear operator on the Banach space $\mathbb{X}$, then $D(L), \rho(L)$, and $\sigma(L)$ stand respectively for the domain, resolvent, and spectrum of $L$. Moreover, one sets $R(\lambda, L):=(\lambda I-L)^{-1}$ for all $\lambda \in \rho(A)$. Furthermore, we set $Q=I-P$ for a projection $P$. We denote the Banach algebra of bounded linear operators on $\mathbb{X}$ by $B(\mathbb{X})$.

Let $B(\mathbb{R}, \mathbb{X})$ stand for the Banach space of all bounded continuous functions $\varphi: \mathbb{R} \mapsto \mathbb{X}$ when equipped with the sup norm $\|\varphi\|_{\infty}:=\sup _{t \in \mathbb{R}}\|\varphi(t)\|$ for $\varphi \in$ $B C(\mathbb{R}, \mathbb{X})$. Similarly, $B\left(\mathbb{R}, \mathbb{X}_{\alpha}\right)$ for $\alpha \in(0,1)$ will stand for the Banach space of all bounded continuous functions $\varphi: \mathbb{R} \mapsto \mathbb{X}_{\alpha}$ when equipped with the $\alpha$-sup norm $\|\varphi\|_{\alpha, \infty}:=\sup _{t \in \mathbb{R}}\|\varphi(t)\|_{\alpha}$ for $\varphi \in B C\left(\mathbb{R}, \mathbb{X}_{\alpha}\right)$. 


\subsection{Almost periodic functions.}

Definition 2.1. A continuous function $f: \mathbb{R} \mapsto \mathbb{X}$ is called (Bohr) almost periodic if for each $\varepsilon>0$ there exists $l(\varepsilon)>0$ such that every interval of length $l(\varepsilon)$ contains a number $\tau$ with the property that

$$
\|f(t+\tau)-f(t)\|<\varepsilon \text { for each } t \in \mathbb{R} .
$$

The number $\tau$ above is called an $\varepsilon$-translation number of $f$, and the collection of all such functions will be denoted $A P(\mathbb{X})$.

Definition 2.2. A continuous function $F: \mathbb{R} \times \mathbb{X} \mapsto \mathbb{X}$ is called (Bohr) almost periodic in $t \in \mathbb{R}$ uniformly in $x \in K$ where $K \subset \mathbb{X}$ is any compact subset if for each $\varepsilon>0$ there exists $l(\varepsilon)$ such that every interval of length $l(\varepsilon)$ contains a number $\tau$ with the property that

$$
\|F(t+\tau, y)-F(t, y)\|<\varepsilon \text { for each } t \in \mathbb{R}, \quad y \in K .
$$

The collection of those functions is denoted by $A P(\mathbb{R}, \mathbb{X})$.

The proof of our main result requires the following composition theorem.

Theorem 2.3. Let $F: \mathbb{R} \times \mathbb{X} \mapsto \mathbb{X}$ be an almost periodic function. Suppose $F(t, u)$ is uniformly continuous on every bounded subset $K^{\prime} \subset \mathbb{X}$ uniformly for $t \in \mathbb{R}$. If $g \in A P(\mathbb{X})$, then $\Gamma: \mathbb{R} \rightarrow \mathbb{X}$ defined by $\Gamma(\cdot):=F(\cdot, g(\cdot))$ belongs to $A P(\mathbb{X})$.

2.2. Evolution families. The setting of this subsection and that on estimates of $U(t, s)$ are similar to that of Diagana [6].

Definition 2.4 (Acquistapace-Terreni conditions [4]). A family of closed linear operators $A(t)$ for $t \in \mathbb{R}$ on $\mathbb{X}$ with domain $D(A(t)$ ) (possibly not densely defined) satisfies the so-called Acquistapace-Terreni conditions. That is, there exist constants $\omega \in \mathbb{R}, \theta \in\left(\frac{\pi}{2}, \pi\right), K, L \geq 0$ and $\mu, \nu \in(0,1]$ with $\mu+\nu>1$ such that

$$
S_{\theta} \cup\{0\} \subset \rho(A(t)-\omega) \ni \lambda, \quad\|R(\lambda, A(t)-\omega)\| \leq \frac{K}{1+|\lambda|}
$$

and

$$
\|(A(t)-\omega) R(\lambda, A(t)-\omega)[R(\omega, A(t))-R(\omega, A(s))]\| \leq L|t-s|^{\mu}|\lambda|^{-\nu}
$$

for $t, s \in \mathbb{R}, \lambda \in S_{\theta}:=\{\lambda \in \mathbb{C} \backslash\{0\}:|\arg \lambda| \leq \theta\}$.

Among other things, the Acquistapace-Terreni conditions ensure that there exists a unique evolution family

$$
\mathcal{U}=\{U(t, s): t, s \in \mathbb{R} \text { such that } t \geq s\}
$$

on $\mathbb{X}$ associated with $A(t)$ such that $U(t, s) \mathbb{X} \subseteq D(A(t))$ for all $t, s \in \mathbb{R}$ with $t \geq s$, and

(a) $U(t, s) U(s, r)=U(t, r)$ for $t, s \in \mathbb{R}$ such that $t \geq s \geq s$;

(b) $U(t, t)=I$ for $t \in \mathbb{R}$, where $I$ is the identity operator of $\mathbb{X}$;

(c) $(t, s) \mapsto U(t, s) \in B(\mathbb{X})$ is continuous for $t>s$.

In that case we say that $A(\cdot)$ generates the evolution family $U(\cdot, \cdot)$.

Definition $2.5(44)$. One says that an evolution family $\mathcal{U}$ has an exponential dichotomy (or is hyperbolic) if there are projections $P(t)(t \in \mathbb{R}$ ) that are uniformly bounded and strongly continuous in $t$ and constants $\delta>0$ and $N \geq 1$ such that

(d) $U(t, s) P(s)=P(t) U(t, s)$; 
(e) the restriction $U_{Q}(t, s): Q(s) \mathbb{X} \rightarrow Q(t) \mathbb{X}$ of $U(t, s)$ is invertible (we then set $\left.\widetilde{U}_{Q}(s, t):=U_{Q}(t, s)^{-1}\right)$; and

(f) $\|U(t, s) P(s)\| \leq N e^{-\delta(t-s)}$ and $\left\|\widetilde{U}_{Q}(s, t) Q(t)\right\| \leq N e^{-\delta(t-s)}$ for $t \geq s$ and $t, s \in \mathbb{R}$.

2.3. Estimates for $U(t, s)$. Let $A$ be a sectorial operator on $\mathbb{X}$ (for that, in Eq. (2.1), replace $A(t)$ with $A$ ) and let $\alpha \in(0,1)$. Define the real interpolation space

$$
\mathbb{X}_{\alpha}^{A}:=\left\{x \in \mathbb{X}:\|x\|_{\alpha}^{A}:=\sup _{r>0}\left\|r^{\alpha}(A-\omega) R(r, A-\omega) x\right\|<\infty\right\},
$$

which, by the way, is a Banach space when endowed with the norm $\|\cdot\|_{\alpha}^{A}$. For convenience we further write $\mathbb{X}_{0}^{A}:=\mathbb{X},\|x\|_{0}^{A}:=\|x\|, \mathbb{X}_{1}^{A}:=D(A)$ and $\|x\|_{1}^{A}:=$ $\|(\omega-A) x\|$. Moreover, let $\hat{\mathbb{X}}^{A}:=\overline{D(A)}$ of $\mathbb{X}$. In particular, we have the following continuous embedding:

$$
D(A) \hookrightarrow \mathbb{X}_{\beta}^{A} \hookrightarrow D\left((\omega-A)^{\alpha}\right) \hookrightarrow \mathbb{X}_{\alpha}^{A} \hookrightarrow \hat{\mathbb{X}}^{A} \hookrightarrow \mathbb{X},
$$

for all $0<\alpha<\beta<1$, where the fractional powers are defined in the usual way.

In general, $D(A)$ is not dense in the spaces $\mathbb{X}_{\alpha}^{A}$ and $\mathbb{X}$. However, we have the following continuous injection $\mathbb{X}_{\beta}^{A} \hookrightarrow \overline{D(A)}\left\|^{\|}\right\|_{\alpha}^{A}$ for $0<\alpha<\beta<1$.

Given the family of linear operators $A(t)$ for $t \in \mathbb{R}$, satisfying (H.1), we set $\mathbb{X}_{\alpha}^{t}:=\mathbb{X}_{\alpha}^{A(t)}$ and $\hat{\mathbb{X}}^{t}:=\hat{\mathbb{X}}^{A(t)}$ for $0 \leq \alpha \leq 1$ and $t \in \mathbb{R}$, with the corresponding norms.

The proofs of the main results of this paper require the following assumptions:

(H.1) The linear operators $\{A(t)\}_{t \in \mathbb{R}}$ satisfy the Acquistapace-Terreni conditions with constants as in Definition 2.4 .

(H.2) The evolution family $\mathcal{U}$ generated by $A(\cdot)$ has an exponential dichotomy with constants $N, \delta>0$ and dichotomy projections $P(t)$ for $t \in \mathbb{R}$. Moreover, $0 \in \rho(A(t))$ for each $t \in \mathbb{R}$ and the following holds:

$$
\sup _{t, s \in \mathbb{R}}\left\|A(s) A^{-1}(t)\right\|_{B\left(\mathbb{X}, \mathbb{X}_{\alpha}\right)}<c_{0} .
$$

(H.3) There exists $0 \leq \alpha<\beta<1$ such that

$$
\mathbb{X}_{\alpha}^{t}=\mathbb{X}_{\alpha} \text { and } \mathbb{X}_{\beta}^{t}=\mathbb{X}_{\beta}
$$

for all $t \in \mathbb{R}$, with uniform equivalent norms.

We have the following fundamental estimates for the evolution family $U(t, s)$.

Proposition 2.6 (4]). Suppose the evolution family $U=U(t, s)$ has exponential dichotomy. For $x \in \mathbb{X}, 0 \leq \alpha \leq 1$ and $t>s$, the following hold:

(i) There is a constant $c(\alpha)$ such that

$$
\|U(t, s) P(s) x\|_{\alpha}^{t} \leq c(\alpha) e^{-\frac{\delta}{2}(t-s)}(t-s)^{-\alpha}\|x\| .
$$

(ii) There is a constant $m(\alpha)$ such that

$$
\left\|\widetilde{U}_{Q}(s, t) Q(t) x\right\|_{\alpha}^{s} \leq m(\alpha) e^{-\delta(t-s)}\|x\| .
$$




\section{Preliminary Results}

Consider the nonautonomous differential equation

$$
\frac{d u}{d t}=A(t) u(t)+F(t, u(t)), \quad t \in \mathbb{R},
$$

where $F: \mathbb{R} \times \mathbb{X}_{\alpha} \mapsto \mathbb{X}$ is jointly continuous.

Definition 3.1. Under assumption (H.1), a function $u: \mathbb{R} \mapsto \mathbb{X}_{\alpha}$ is said to be a mild solution to Eq. (3.1) provided that

$$
u(t)=U(t, s) u(s)+\int_{s}^{t} U(t, \tau) F(\tau, u(\tau)) d \tau
$$

for each $\forall t \geq s, t, s \in \mathbb{R}$.

It is not difficult to see that if the function $F: \mathbb{R} \times \mathbb{X}_{\alpha} \mapsto \mathbb{X}$ is bounded, then

$$
u(t)=\int_{-\infty}^{t} U(t, s) P(s) F(s, u(s)) d s-\int_{t}^{\infty} U_{Q}(t, s) Q(s) F(s, u(s)) d s,
$$

for all $t \in \mathbb{R}$, is a mild solution to Eq. (3.1).

Throughout the rest of the paper we fix $\alpha, \beta$, real numbers satisfying $0<\alpha<$ $\beta<1$ with $2 \beta>\alpha+1$.

To study the existence of almost periodic solutions to Eq. (3.1), in addition to the previous assumptions, we suppose that the injection

$$
\mathbb{X}_{\alpha} \hookrightarrow \mathbb{X}
$$

is compact and that the following additional assumptions hold:

(H.4) $R(\omega, A(\cdot)) \in A P\left(B\left(\mathbb{X}, \mathbb{X}_{\alpha}\right)\right)$.

(H.5) (a) The function $F: \mathbb{R} \times \mathbb{X}_{\alpha} \mapsto \mathbb{X}$ is almost periodic in the first variable and uniformly in the second one. The function $u \mapsto F(t, u)$ is uniformly continuous on any bounded subset $K$ of $\mathbb{X}_{\alpha}$ for each $t \in \mathbb{R}$. Finally,

$$
\|F(t, u)\|_{\infty} \leq \mathcal{M}\left(\|u\|_{\alpha, \infty}\right)
$$

where $\mathcal{M}: \mathbb{R}^{+} \mapsto \mathbb{R}^{+}$is a continuous, monotone increasing function satisfying

$$
\lim _{r \rightarrow \infty} \frac{\mathcal{M}(r)}{r}=0 .
$$

We denote by $S=S_{1}-S_{2}$ the nonlinear integral operators $S_{1}$ and $S_{2}$ defined by

$$
\left(S_{1} u\right)(t):=\int_{-\infty}^{t} U(t, s) P(s) F(s, u(s)) d s
$$

and

$$
\left(S_{2} u\right)(t):=\int_{t}^{\infty} U_{Q}(t, s) Q(s) F(s, u(s)) d s .
$$

The proof of the main result of the present paper requires the next lemma.

Lemma 3.2 ([6, Diagana]). For each $x \in \mathbb{X}$, suppose that assumptions (H.1)-(H.2) hold and let $\alpha, \beta$ be real numbers such that $0<\alpha<\beta<1$ with $2 \beta>\alpha+1$. Then there are two constants $r^{\prime}(\alpha, \beta), d^{\prime}(\beta)>0$ such that

$$
\|A(t) U(t, s) P(s) x\|_{\beta} \leq r^{\prime}(\alpha, \beta) e^{-\frac{\delta}{4}(t-s)}(t-s)^{-\beta}\|x\|, \quad t>s,
$$


and

$$
\left\|A(t) \widetilde{U}_{Q}(t, s) Q(s) x\right\|_{\beta} \leq d^{\prime}(\beta) e^{-\delta(s-t)}\|x\|, \quad t \leq s .
$$

Lemma 3.3 ([6, Diagana]). Under assumptions (H.1)-(H.3), the mapping $S$ : $B C\left(\mathbb{R}, \mathbb{X}_{\alpha}\right) \mapsto B C\left(\mathbb{R}, \mathbb{X}_{\alpha}\right)$ is well-defined and continuous.

Lemma 3.4 ([6, Diagana]). Under assumptions (H.1)-(H.5), $S=S_{1}-S_{2}$ maps bounded sets of $B C\left(\mathbb{R}, \mathbb{X}_{\alpha}\right)$ into bounded sets of $B C^{\gamma}\left(\mathbb{R}, \mathbb{X}_{\alpha}\right)$ for some $0<\gamma<1$, where $S_{1}, S_{2}$ are the integral operators introduced previously.

The proof of the next lemma follows along the same lines as that of Lemma 3.4 and is hence omitted.

Lemma 3.5. The integral operator $S=S_{1}-S_{2}$ maps bounded sets of $A P\left(\mathbb{X}_{\alpha}\right)$ into bounded sets of $B C^{1-\beta}\left(\mathbb{R}, \mathbb{X}_{\alpha}\right) \cap A P\left(\mathbb{X}_{\alpha}\right)$.

Similarly, the next lemma is a consequence of [7, Proposition 3.3].

Lemma 3.6. The set $B C^{1-\beta}\left(\mathbb{R}, \mathbb{X}_{\alpha}\right)$ is compactly contained in $B C(\mathbb{R}, \mathbb{X})$; that is, the canonical injection id : $B C^{1-\beta}\left(\mathbb{R}, \mathbb{X}_{\alpha}\right) \mapsto B C(\mathbb{R}, \mathbb{X})$ is compact, which yields that

is compact, too.

$$
i d: B C^{1-\beta}\left(\mathbb{R}, \mathbb{X}_{\alpha}\right) \cap A P\left(\mathbb{X}_{\alpha}\right) \mapsto A P\left(\mathbb{X}_{\alpha}\right)
$$

Theorem 3.7. Suppose assumptions (H.1)-(H.5) hold. Then Eq. (3.1) has an almost periodic mild solution.

Proof. The proof follows along the same lines as that of [6, Theorem 3.8]. For the sake of clarity, we reproduce it here. Let us recall that in view of Lemma 3.4 we have

$$
\|S u\|_{\alpha, \infty} \leq p(\alpha, \beta, \delta) \mathcal{M}\left(\|u\|_{\alpha, \infty}\right)
$$

and

$$
\left\|S u\left(t_{2}\right)-S u\left(t_{1}\right)\right\|_{\alpha} \leq s(\alpha, \beta, \delta) \mathcal{M}\left(\|u\|_{\alpha, \infty}\right)\left|t_{2}-t_{1}\right|
$$

for all $u \in B C\left(\mathbb{R}, \mathbb{X}_{\alpha}\right), t_{1}, t_{2} \in \mathbb{R}$ with $t_{1} \neq t_{2}$, where $p(\alpha, \beta, \delta)$ and $s(\alpha, \beta, \delta)$ are positive constants. Consequently, $u \in B C\left(\mathbb{R}, \mathbb{X}_{\alpha}\right)$ and $\|u\|_{\alpha, \infty}<R$ yield $S u \in$ $B C^{1-\beta}\left(\mathbb{R}, \mathbb{X}_{\alpha}\right)$ and $\|S u\|_{\alpha}<R_{1}$, where $R_{1}=c(\alpha, \beta, \delta) \mathcal{M}(R)$.

Therefore, there exists $r>0$ such that for all $R \geq r$, the following holds:

$$
S\left(B_{A P\left(\mathbb{X}_{\alpha}\right)}(0, R)\right) \subset B_{B C^{1-\beta}\left(\mathbb{R}, \mathbb{X}_{\alpha}\right)}(0, R) \cap B_{A P\left(\mathbb{X}_{\alpha}\right)}(0, R) .
$$

In view of the above, it follows that $S: D \mapsto D$ is continuous and compact, where $D$ is the ball in $A P\left(\mathbb{X}_{\alpha}\right)$ of radius $R$ with $R \geq r$. Using the Schauder fixed point theorem it follows that $S$ has a fixed point, which obviously is an almost periodic mild solution to Eq. (3.1).

\section{Almost periodic solutions to Eq. (1.2)}

Let $\mathbb{H}$ be an infinite dimensional separable complex Hilbert space equipped with the norm $\|\cdot\|$. Let $\left\{e_{j}^{k}\right\}$ be a (complete) orthonormal sequence of eigenvectors associated with the eigenvalues $\left\{\lambda_{j}\right\}_{j \geq 1}$. Therefore, for each

$$
u \in D(A):=\left\{u \in \mathbb{H}: \sum_{j=1}^{\infty} \lambda_{j}^{2}\left\|E_{j} u\right\|^{2}<\infty\right\},
$$


then

$$
A u=\sum_{j=1}^{\infty} \lambda_{j} \sum_{k=1}^{\gamma_{j}}\left\langle u, e_{j}^{k}\right\rangle e_{j}^{k}=\sum_{j=1}^{\infty} \lambda_{j} E_{j} u
$$

where $E_{j} u=\sum_{k=1}^{\gamma_{j}}\left\langle u, e_{j}^{k}\right\rangle e_{j}^{k}$

In this section, we take $\mathbb{X}=\mathbb{H}^{2}=\mathbb{H} \times \mathbb{H}$ equipped with its natural topology. To study the existence of almost periodic solutions to Eq. (1.2), in addition to the previous assumptions, we suppose that the injection

$$
\mathbb{H}_{\alpha} \hookrightarrow \mathbb{H}
$$

is compact and that the following additional assumption holds:

(H.6) The function $f, g: \mathbb{R} \times \mathbb{H}_{\alpha} \mapsto \mathbb{H}$ is almost periodic in the first variable and uniformly in the second one. The functions $u \mapsto f(t, u)$ and $u \mapsto g(t, u)$ are uniformly continuous on any bounded subset $K$ of $\mathbb{H}_{\alpha}$ for each $t \in \mathbb{R}$. Finally,

$$
\left(\|f(t, u)\|_{\infty}^{2}+\|g(t, u)\|_{\infty}^{2}\right)^{1 / 2} \leq \mathcal{M}\left(\|u\|_{\alpha, \infty}\right)
$$

for all $u \in \mathbb{H}_{\alpha}$, where $\mathcal{M}: \mathbb{R}^{+} \mapsto \mathbb{R}^{+}$is a continuous, monotone increasing function satisfying

$$
\lim _{r \rightarrow \infty} \frac{\mathcal{M}(r)}{r}=0
$$

Theorem 4.1. Under previous assumptions and if (H.6) holds, then Eq. (1.2) has at least one almost periodic solution $u \in \mathbb{H}_{\alpha}$.

Proof. For all $z:=\left(\begin{array}{l}u \\ v\end{array}\right) \in D=D(\mathcal{A}(t))=D(A) \times \mathbb{H}$, we obtain the following:

$$
\mathcal{A}(t) z=\sum_{n=1}^{\infty} \mathcal{A}_{n}(t) P_{n} z
$$

where $I_{\mathbb{H}}$ is the identity operator of $\mathbb{H}$,

$$
P_{n}:=\left(\begin{array}{cc}
E_{n} & 0 \\
0 & E_{n}
\end{array}\right), n \geq 1
$$

and

$$
\mathcal{A}_{n}(t):=\left(\begin{array}{cc}
0 & 1 \\
w(t) \lambda_{n} & 0
\end{array}\right), n \geq 1, \quad t \in \mathbb{R} .
$$

Now, the characteristic equation for $\mathcal{A}_{n}(t)$ is given by

$$
\lambda^{2}-\lambda_{n} w(t)=\lambda^{2}-\lambda_{n} \rho(t) e^{i \theta(t)}=0,
$$

from which we obtain its eigenvalues given by

$$
\begin{aligned}
\lambda_{1}^{n}(t) & =\sqrt{\lambda_{n} \rho(t)} e^{i \frac{\theta(t)}{2}} \text { and } \lambda_{2}^{n}(t)=\sqrt{\lambda_{n} \rho(t)} e^{-i \frac{\theta(t)}{2}}, \\
\text { and hence } \sigma\left(\mathcal{A}_{n}(t)\right) & =\left\{\lambda_{1}^{n}(t), \lambda_{2}^{n}(t)\right\} .
\end{aligned}
$$


Noticing that $\mathcal{A}(t)$ is invertible for all $t \in \mathbb{R}$ and using Eq. (1.3), it follows that there exists $\theta \in\left(\frac{\pi}{2}, \pi\right)$ such that $S_{\theta} \cup\{0\} \subset \rho(\mathcal{A}(t))$.

On the other hand, since $\lambda_{1}^{n}$ and $\lambda_{2}^{n}$ are distinct, $\mathcal{A}_{n}(t)$ is diagonalizable. Further, it is not difficult to see that $\mathcal{A}_{n}(t)=K_{n}^{-1}(t) J_{n}(t) K_{n}(t)$, where $J_{n}(t), K_{n}(t)$ and $K_{n}^{-1}(t)$ are respectively given by

$$
J_{n}(t)=\left(\begin{array}{cc}
\lambda_{1}^{n}(t) & 0 \\
0 & \lambda_{2}^{n}(t)
\end{array}\right), \quad K_{n}(t)=\left(\begin{array}{cc}
1 & 1 \\
\lambda_{1}^{n}(t) & \lambda_{2}^{n}(t)
\end{array}\right),
$$

and

$$
K_{n}^{-1}(t)=\frac{1}{\lambda_{1}^{n}(t)-\lambda_{2}^{n}(t)}\left(\begin{array}{cc}
-\lambda_{2}^{n}(t) & 1 \\
\lambda_{1}^{n}(t) & -1
\end{array}\right) .
$$

For $\lambda \in S_{\theta}$ and $z \in \mathbb{X}$, one has

$$
\begin{aligned}
R(\lambda, \mathcal{A}(t)) z & =\sum_{n=1}^{\infty}\left(\lambda-\mathcal{A}_{n}(t)\right)^{-1} P_{n} z \\
& =\sum_{n=1}^{\infty} K_{n}(t)\left(\lambda-J_{n}(t)\right)^{-1} K_{n}^{-1}(t) P_{n} z
\end{aligned}
$$

It can be shown that

$$
\|R(\lambda, \mathcal{A}(t))\| \leq \frac{K}{1+|\lambda|}
$$

for all $\lambda \in S_{\theta}$ and $t \in \mathbb{R}$.

Now, for every $t \in \mathbb{R}$, the family of linear operators $\mathcal{A}(t)$ generate an analytic semigroup $\left(e^{\tau \mathcal{A}(t)}\right)_{\tau \geq 0}$ on $\mathbb{X}$ given by

$$
e^{\tau \mathcal{A}(t)} z=\sum_{n=0}^{\infty} K_{n}(t)^{-1} P_{n} e^{\tau J_{n}} P_{n} K_{n}(t) P_{n} z, z \in \mathbb{X} .
$$

On the other hand, we have

$$
\left\|e^{\tau \mathcal{A}(t)} z\right\|=\sum_{n=0}^{\infty}\left\|K_{n}(t)^{-1} P_{n}\right\|\left\|e^{\tau J_{n}} P_{n}\right\|\left\|K_{n}(t) P_{n}\right\|\left\|P_{n} z\right\|,
$$

with for each $z=\left(\begin{array}{c}z_{1} \\ z_{2}\end{array}\right)$

$$
\begin{aligned}
\left\|e^{\tau J_{n}} P_{n} z\right\|^{2} & =\left\|\left(\begin{array}{cc}
e^{\lambda_{1}^{n}(t) \tau} E_{n} & 0 \\
& \\
0 & e^{\lambda_{2}^{n}(t) \tau} E_{n}
\end{array}\right)\left(\begin{array}{c}
z_{1} \\
z_{2}
\end{array}\right)\right\|^{2} \\
& \leq\left\|e^{\lambda_{1}^{n}(t) \tau} E_{n} z_{1}\right\|^{2}+\left\|e^{\lambda_{2}^{n}(t) \tau} E_{n} z_{2}\right\|^{2} \\
& \leq e^{2 \Re e\left(\lambda_{1}^{n}(t)\right) \tau}\|z\|^{2} .
\end{aligned}
$$


Clearly, using Eq. (1.3) it follows that

$$
\begin{aligned}
\Re e\left(\lambda_{1}^{n}(t)\right) & =\sqrt{\lambda_{n} \rho(t)} \cos \left(\frac{\theta(t)}{2}\right) \\
& \leq \sqrt{\lambda_{1} \rho_{0}} \cos \left(\frac{\theta(t)}{2}\right) \\
& \leq \sqrt{\lambda_{1} \rho_{0}} \cos \left(\frac{\pi}{2}+\theta_{0}\right) \\
& =-\sqrt{\lambda_{1} \rho_{0}} \sin \left(\theta_{0}\right) .
\end{aligned}
$$

Setting $\delta=\sqrt{\lambda_{1} \rho_{0}} \sin \left(\theta_{0}\right)>0$, it follows that there exists $C_{0}>0$ such that

$$
\left\|e^{\tau \mathcal{A}(t)}\right\| \leq C_{0} e^{-\delta \tau}, \quad \tau \geq 0 .
$$

Therefore, the evolution family $(U(t, s))_{t \geq s}$ is exponentially stable.

Clearly, assumptions (H.2) and (H.3) hold. It remains to check assumption (H.4). Now since $t \mapsto w(t)$ and $t \mapsto w(t)^{-1}$ are almost periodic it follows that $t \mapsto \mathcal{A}(t)^{-1}$ is almost periodic with respect to operator topology. Using Theorem 3.7 it follows that Eq. (1.2) has at least one almost periodic mild solution.

\section{Existence of almost periodic solutions to Eq. (1.1)}

In order to study Eq. (1.1) we still suppose that the injection $\mathbb{H}_{\alpha} \hookrightarrow \mathbb{H}$ is compact and that the following additional assumptions hold:

$\left(h_{1}\right)$ The function $b: \mathbb{R} \mapsto \mathbb{C}$ is given such that $b(t)=-\widetilde{\rho}(t) e^{i \widetilde{\theta}(t)}$ for all $t \in \mathbb{R}$ is almost periodic and there exist $\widetilde{\rho}_{0}, \widetilde{\rho}_{1}>0$ and $\widetilde{\theta}_{0} \in\left(0, \frac{\pi}{4}\right)$ such that $\widetilde{\rho}_{0} \leq \widetilde{\rho}(t) \leq \widetilde{\rho}_{1}$, and $\pi+2 \widetilde{\theta}_{0}<\widetilde{\theta}(t)<2 \pi-2 \widetilde{\theta}_{0}$ for all $t \in \mathbb{R}$.

$\left(h_{2}\right)$ There exist $L_{0}>0$ and $\mu \in(0,1]$ such that $|b(t)-b(s)| \leq L_{0}|t-s|^{\mu}$ for all $s, t \in \mathbb{R}$.

$\left(h_{3}\right)$ The function $a: \mathbb{R} \mapsto \mathbb{C}$ is uniformly continuous, almost periodic, and differentiable.

$\left(h_{4}\right) h: \mathbb{R} \times \mathbb{H} \mapsto \mathbb{H}$ is almost periodic in the first variable uniformly in the second one. The function $u \mapsto h(t, u)$ is uniformly continuous on any bounded subset $K^{\prime}$ of $\mathbb{H}_{\alpha}$ for each $t \in \mathbb{R}$. Finally,

$$
\left(2\|h(t, u)\|_{\infty}^{2}+3 k^{2} m^{2}\|u\|_{\alpha, \infty}^{2}\right)^{1 / 2} \leq \mathcal{Q}\left(\|u\|_{\alpha, \infty}\right)
$$

for all $u \in \mathbb{H}_{\alpha}$, where $k$ is the bound of the injection $\mathbb{H}_{\alpha} \hookrightarrow \mathbb{H}, m:=$ $\max \left(\|a\|_{\infty},\left\|a^{\prime}\right\|_{\infty}\right)$, and $\mathcal{Q}: \mathbb{R}^{+} \mapsto \mathbb{R}^{+}$is a continuous, monotone increasing function satisfying

$$
\lim _{r \rightarrow \infty} \frac{\mathcal{Q}(r)}{r}=0 .
$$

Theorem 5.1. Under previous assumptions and if $\left(h_{1}\right)-\left(h_{4}\right)$ hold, then Eq. (1.1) has at least one almost periodic solution $u \in \mathbb{H}_{\alpha}$.

Proof. It suffices to let $w(t)=-b(t), g(t, u)=a(t) u$, and $f(t, u)=h(t, u)+u a^{\prime}(t)$ for all $t \in \mathbb{R}$ in Eq. (1.2) and to make use of Theorem 4.1 


\section{EXAMPLE}

Let $\Omega \subset \mathbb{R}^{N}(N \geq 1)$ be an open bounded subset with $C^{2}$ boundary $\partial \Omega$ and let $\mathbb{H}=L^{2}(\Omega)$ be equipped with its natural topology.

To illustrate our abstract results, we study the existence of almost periodic solutions to the following damped second-order boundary value problem:

$$
\begin{gathered}
\frac{\partial^{2} u}{\partial t^{2}}+a(t, x) \frac{\partial u}{\partial t}-b(t, x) \Delta u=h(t, x, u), \quad t \in \mathbb{R}, \quad x \in \Omega, \\
u(t, x)=0, \quad t \in \mathbb{R}, \quad x \in \partial \Omega
\end{gathered}
$$

where $a: \mathbb{R} \times \Omega \mapsto \mathbb{C}$ satisfies $\left(h_{3}\right), b: \mathbb{R} \times \Omega \mapsto \mathbb{C}$ satisfies $\left(h_{1}\right)-\left(h_{2}\right)$, and $h$ : $\mathbb{R} \times \Omega \times L^{2}(\Omega) \mapsto L^{2}(\Omega)$ satisfies $\left(h_{4}\right)$.

Define the linear operator $A$ as follows:

$$
A u=-\Delta u \text { for all } u \in D(A)=H_{0}^{1}(\Omega) \cap H^{2}(\Omega) .
$$

Let $\alpha \in(0,1)$ and $q \in(0, \infty]$. Using the fact that the injection $H_{0}^{1}(\Omega) \hookrightarrow L^{2}(\Omega)$ is compact, it follows that $H_{0}^{1}(\Omega) \cap H^{2}(\Omega) \hookrightarrow L^{2}(\Omega)$ is compact, too. It can then be shown that the injection $\left(L^{2}(\Omega), H_{0}^{1}(\Omega) \cap H^{2}(\Omega)\right)_{\alpha, q} \hookrightarrow L^{2}(\Omega)$ is compact, where the space $\left(L^{2}(\Omega), H_{0}^{1}(\Omega) \cap H^{2}(\Omega)\right)_{\alpha, q}$ is the real interpolation space of order $(\alpha, q)$ between $L^{2}(\Omega)$ and $H_{0}^{1}(\Omega) \cap H^{2}(\Omega)$. Therefore, letting $q=\infty$, it follows that the injection $\mathbb{H}_{\alpha}=\left(L^{2}(\Omega), H_{0}^{1}(\Omega) \cap H^{2}(\Omega)\right)_{\alpha, \infty} \hookrightarrow L^{2}(\Omega)$ is compact.

Consequently, taking into account the previous assumptions, it follows that the system Eq. (6.1)-Eq. (6.2) has at least one almost periodic mild solution.

\section{REFERENCES}

1. K. Balachandran and S. Marshal Anthoni, Controllability of second-order semilinear neutral functional differential systems in Banach spaces. Comput. Math. Appl. 41 (2001), no. 10-11, 1223-1235. MR1824921 (2002b:93012)

2. K. Balachandran and S. Marshal Anthoni, Existence of solutions of second-order neutral functional differential equations. Tamkang J. Math. 30 (1999), no. 4, 299-309. MR.1729273 (2000m:34181)

3. M. Benchohra, J. Henderson, and S. K. Ntouyas, Existence results for impulsive multivalued semilinear neutral functional differential inclusions in Banach spaces, J. Math. Anal. Appl. 236 (2001), no. 2, 763-780. MR.1866239(2002h:34162)

4. M. Baroun, S. Boulite, T. Diagana, and L. Maniar, Almost periodic solutions to some semilinear non-autonomous thermoelastic plate equations. J. Math. Anal. Appl. 349 (2009), no. 1, 74-84. MR2455732 (2010a:35253)

5. C. Corduneanu, Almost periodic functions, 2nd edition, Chelsea, New York, 1989.

6. T. Diagana, Almost automorphic mild solutions to some classes of nonautonomous higherorder differential equations. Semigroup Forum. DOI: 10.1007/s00233-010-9261-y.

7. J. A. Goldstein and G. M. N'Guérékata, Almost automorphic solutions of semilinear evolution equations. Proc. Amer. Math. Soc. 133 (2005), no. 8, 2401-2408. MR2138883 (2006a:34175)

8. E. M. Hernández, Existence of solutions to a second-order partial differential equation with nonlocal conditions. Electronic J. Diff. Eqns. 2003 (2003), no. 51, 1-10. MR1971117 (2004a:34101)

9. H. Leiva, Existence of bounded solutions of a second-order system with dissipation. J. Math. Anal. Appl. 237 (1999), 288-302. MR1708174 (2000g:34040)

10. A. Lunardi, Analytic semigroups and optimal regularity in parabolic problems. Progress in Nonlinear Diff. Eqns. and Their Applications, Vol. 16, Birkhäuser Verlag, Basel, 1995. MR 1329547 (96e:47039) 
11. S. K. Ntouyas, Global existence results for certain second order delay integrodifferential equations with nonlocal conditions. Dynam. Systems Appl. 7 (1998), no. 3, 415-425. MR 1639604 (99h:45013)

12. S. K. Ntouyas and P. Ch. Tsamatos, Global existence for second order semilinear ordinary and delay integrodifferential equations with nonlocal conditions. Appl. Anal. 67 (1997), no. 3-4, 245-257. MR1614061 (98k:34103)

13. S. K. Ntouyas and P. Ch. Tsamatos, On the solvability of a boundary value problem for a second order differential equation with deviating arguments. Commun. Appl. Anal. 1 (1997), no. 4, 525-538. MR.1470819 (99d:34122)

14. T. J. Xiao, J. Liang, The Cauchy problem for higher-order abstract differential equations, Lecture Notes in Mathematics, Vol. 1701, Springer, Berlin, 1998. MR1725643 (2001a:34099)

Department of Mathematics, Howard University, 2441 6th Street N.W., Washington, DC 20059

E-mail address: tdiagana@howard.edu 